\title{
Editorial
}

\section{Perceived Elementary Grid (PEG) proposal for dyadic coping evaluation in the infertile couple during assisted reproductive treatments}

\author{
Giovanni Buzzaccarini ${ }^{1, *}$, Amerigo Vitagliano ${ }^{1}$, Andrea Busnelli $^{2}$, Marco Noventa $^{1}$, \\ Loris Marin $^{1}$, Antonio Simone Laganà ${ }^{3}$, Giuseppe Gullo ${ }^{4}$, Marco Inghilleri ${ }^{5}$, \\ Simona Luciani ${ }^{5}$, Guido Ambrosini ${ }^{1}$, Alessandra Andrisani ${ }^{1}$ \\ ${ }^{1}$ Department of Women and Children's Health, University of Padua, 35128 Padua, Italy \\ ${ }^{2}$ Department of Gynecology, Division of Gynecology and Reproductive Medicine, Fertility Center, Humanitas Clinical and Research Center-IRCCS, \\ Rozzano, 20089 Milan, Italy \\ ${ }^{3}$ Department of Obstetrics and Gynecology, "Filippo Del Ponte" Hospital, University of Insubria, 21100 Varese, Italy \\ ${ }^{4}$ Department of Obstetrics and Gynecology, Villa Sofia Cervello Hospital, University of Palermo, 90133 Palermo, Italy \\ ${ }^{5}$ Interattivamente, Center for Legal Psychology-Clinical Sexology-Psicoterapy, 35139 Padova, Italy \\ *Correspondence: giovanni.buzzaccarini@gmail.com (Giovanni Buzzaccarini) \\ Academic Editor: Michael H. Dahan \\ Submitted: 20 August 2021 Accepted: 7 September 2021 Published: 9 February 2022
}

Infertility is a condition affecting up to $15 \%$ of couples, which may require the intervention of assisted reproductive techniques (ART) [1]. In these couples, both female and male psychological wellbeing is strongly affected by the prolonged and exhausting infertility path [2].

Although various authors have been studying the possible implications of psychological distress in ART outcomes (i.e., clinical pregnancy rates and live birth rates), new insights have been brought on concerning the couple health and wellbeing [3]. Accordingly, infertility may be a cause of distress because it results in an inability to fulfil traditional roles. Indeed, those who adhere to traditional sex roles may be more hampered by the experience of infertility $[4,5]$.

On this scenario, we aimed to investigate the couple strategy about coping. In particular, the relationship between partners is heavily stressed due to the tightened steps required from ART. Infertility, a struggling event, forces both the female and male partner to fight with undesired and unpleasant diagnosis, which gains more importance if addressed mainly to one partner. In this case, a sense of guilty can affect one partner and strongly hamper his/her wellbeing. Conversely, dyadic coping expresses the couple ability to deal with unforeseen events, such as infertility [6]. This type of coping, which has been widely studied, arises from the strategy in dealing with daily, stressful, and prolonged "icta". However, both female and male partner give their contribution, and, between them, different hierarchical, addictive, and outdistancing relationships occur. Here we present a method of analysis called Perceived Elementary Grid (PEG). Arising from the constructivist psychology, the qualitative grids have been extensively described in their methodology by Procter [7]. In particular, qualitative grids have been developed in the last years as a flexible method of eliciting and displaying interpersonal construing.
The PEG is a graphic table which is filled with piece of information deriving from an interview with each member of the couple. In particular, the PEG explores in a wide and peculiar way the deepest issues regarding the relationships arising from one specific topic. This table examines the way in which a set of characters construe themselves and each of the others. In our specialty, we aimed to apply this method to the male and female partner handling infertility. Our suggestion relies on interviewing both the female and male partner individually, inviting them to consider infertility from different points of view. In this scenario, the consultant should focus on the patient logical and rational discussions, on emotional and sentimental answers, on pose and gestures, and on external referrals. Moreover, we coined a possible way of intervention which finds its key point in a multi-level interpersonal construing (Fig. 1). Specifically, we act in a three-step approach, both for the female and male partner. There are two levels of abstraction: in the first level, the patient should imagine having a different role by construing a multi-level interaction; in the second level, the patient should imagine thinking what opinion he/she would express, given that role.

We first ask one of the two partner to pose him/herself from the perspector eye of him/herself(role 1, monadic). In this case, the patient is construing one person relationship (monadic). Then, we propose to try to focus on issues regarding her/himself and the partner point of view. Finally, we invite the patient to imagine the point of view of "the couple", as a unique thought deriving from both the partners opinion. The main argument of this thinking should be the infertility affecting the couple, although declined in its various facets.

Secondly, we invite the same patient to construe a twoperson relationship (role 2, dyadic) and try to focus on new insights deriving from the same argument. This level in- 


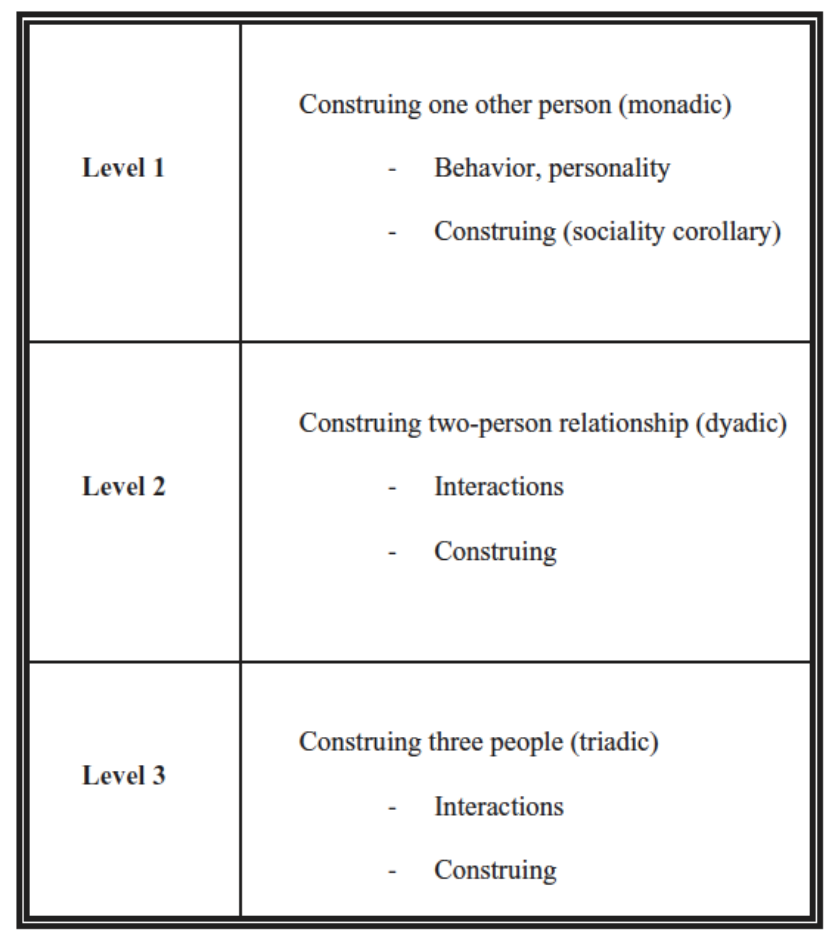

Fig. 1. The multi-level interpersonal construing.

cludes all the construing we apply to the "ourselves" category. In particular, the patients should always focus on his/her own point of view, on the partner opinion, and on the couple possible answers to the issue. The main difference from the first step relies on the fact that all the reasoning is brought on also through the consideration of the partner lenses.

The third and final step requires for the patient the ability to abstract from his/her position and imagine a relationship arising from both the partner perspections (role 3 , triadic). Triadic construction can be seen as a species of Thirdness involving the apprehension of more elements. More specifically, the patient should imagine the couple as one single entity capable of individual reasoning and judgement. Once achieved, the patient is invited to complete the possible scenarios of how her/his and the partner approach to infertility is perceived. Finally, the couple itself is asked to think about the couple ability to deal with infertility.

Graphically, the PEG can be drawn as a $3 \times 3$ grid, where rows express the perspector point of view (the three roles) and columns present the opinions and insights about the three different relations (her/himself, the partner, the couple).

Although complex at first sight, this multi-dyadic approach tries to investigate the deepest aspects of the relationship of the couple and its ability to struggle against the infertility related stress and malaise. When the patient is asked to play a role, new insights and clues are provided which can widen the dyadic analysis of the couple. As a triad, in an ascending climax of abstraction and difficulty, this method could offer the consultant the possi- bility to elicit covered coping resources. Moreover, since both the partners are interviewed and invited to think at the other partner point of view, incongruencies and discrepancies come to surface.

We believe that this approach could be suitable for physicians and psychologists dealing with dyadic coping in the infertile population. In particular, the novelty of this approach linked to the prominent need for care of the infertile patient in his psychological wellbeing could provide new targets of intervention, under the aegis of a tailored medicine.

\section{Author contributions}

All the authors conform the Journal and the International Committee of Medical Journal Editors (ICMJE) criteria for authorship, contributed to the intellectual content of the study and gave approval for the final version of the article. In particular, $\mathrm{GB}, \mathrm{AV}$ and $\mathrm{AB}$ wrote the manuscript; $\mathrm{GB}, \mathrm{MN}, \mathrm{ASL}$, and AA revised it in the final form; LM, GG, and GA provided extraction of data from the literature; MI and SL provided intellectual support regarding the psychological analysis. All the authors approved the manuscript in its final form.

\section{Ethics approval and consent to participate}

Not applicable.

\section{Acknowledgment}

Not applicable.

\section{Funding}

This research received no external funding.

\section{Conflict of interest}

The authors declare no conflict of interest. GB, AV and $\mathrm{AB}$ are the Guest Editor of this journal. MN, ASL, AA are the Editorial Editor of this journal. Given their role as Guest and Editorial board member, they had no involvement in the peer review of this article and has no access to information regarding its peer-review.

\section{References}

[1] Inhorn MC, Patrizio P. Infertility around the globe: new thinking on gender, reproductive technologies and global movements in the 21 st century. Human Reproduction Update. 2015; 21: 411426.

[2] Ragni G, Mosconi P, Baldini MP, Somigliana E, Vegetti W, Caliari I, et al. Health-related quality of life and need for IVF in 1000 Italian infertile couples. Human Reproduction. 2005; 20: $1286-1291$.

[3] Matthiesen SMS, Frederiksen Y, Ingerslev HJ, Zachariae R. Stress, distress and outcome of assisted reproductive technology (ART): a meta-analysis. Human Reproduction. 2011; 26: 2763 2776.

[4] Cook R. The relationship between sex role and emotional func- 
tioning in patients undergoing assisted conception. Journal of Psychosomatic Obstetrics \& Gynecology. 1993; 14: 31-40.

[5] Andrews FM, Abbey A, Halman LJ. Is fertility-problem stress different? The dynamics of stress in fertile and infertile couples. Fertility and Sterility. 1992; 57: 1247-1253.

[6] Molgora S, Fenaroli V, Acquati C, De Donno A, Baldini MP,
Saita E. Examining the Role of Dyadic Coping on the Marital Adjustment of Couples Undergoing Assisted Reproductive Technology (ART). Frontiers in Psychology. 2019; 10: 415.

[7] Procter H. Qualitative Grids, the Relationality Corollary and the Levels of Interpersonal Construing. Journal of Constructivist Psychology. 2014; 27: 243-262. 